\title{
Analysis and Design of TCM Health Management System for Treating End-disease
}

\author{
Gan Xinyan, Gao Xiang, Lan Tianying ${ }^{\text {a. * }}$ \\ Guangxi university of chinese medicine, Nanning, Guangxi 530200, China \\ alantianying@126.com
}

Keywords: "Internet", Treatment of terminal diseases, Health management system.

\begin{abstract}
The 13th Five-Year Plan of the State proposed that by 2020, 30 percent of maternal and child health institutions will provide end-care services and 50 percent of village health clinics will carry out health intervention services with traditional Chinese medicine. In order to achieve this goal, we must resort to "Internet" technology. Therefore, it is urgent for most service organizations to set up a health management system for treating terminal diseases in TCM. This paper discusses how to construct a health management system for treating terminal diseases in hospitals and community. In order to solve some problems encountered in practical use, some discussion and analysis are forward on the key technologies used in this paper.
\end{abstract}

\section{Introduction}

Signed and approved by Premier Li Keqiang, the State Council issued the outline of the Strategic Plan for the Development of traditional Chinese Medicine (2016-2030) in February 2016, which defines the key tasks for the development of traditional Chinese medicine in the coming period: first, it is necessary to improve the medical service capacity of traditional Chinese medicine. Improve the coverage of urban and rural traditional Chinese medicine service network; promote the "Internet" Chinese medicine. By 2020, 30 percent of the maternal and child health institutions will provide services to prevent and cure the disease, and 50 percent of the township health centers and all community service agencies will carry out health intervention services with traditional Chinese medicine. In order to achieve these goals, we must with the help of the Internet, we use the "Internet" technology to build a "Internet" TCM management system for the treatment of pre-illness health that integrates health management, chronic prevention, and other health files. The new model of "diagnosis and treatment in the cloud, doctors around-service in the palm-not ill in supervision" is the mode to be popularized now and in the future. Therefore, it is the trend of the times to study the health management system of TCM.

"Preventing disease" is the basic principle of the theory of TCM treatment, one of the core concepts of TCM, and also an important theoretical basis and criterion of TCM prevention and health care. Jing >:"Upper treatment of disease, Chinese medicine treatment of disease, under the treatment of disease already" [1], that is, the most intelligent doctor treatment has not yet occurred disease, medium doctor treatment will occur disease, and ordinary doctor treatment has occurred disease. Information collection system, however, is not detailed, there is no in-hospital diagnosis and treatment information, and there is a lack of traditional Chinese medicine for the treatment of patients with disease at home medicine, habits, harmful factors prevention and other important data. There are many differences between traditional Chinese medicine and other Western medicine in the treatment of end-of-illness. Most of the health management systems used in the society are just for the community, family-based, and personal health-based. Moreover, these systems are independent of each other and cannot be shared with hospital data, let alone integrated and integrated. To verify, it is difficult to effectively use and in-depth analysis. This system is mainly based on the use of an end care center in a third grade A hospital, and is solved according to the problems actually arising in the course of hospital use, so as to build a suitable hospital and community. 


\section{System overall objective}

\subsection{Overall objectives}

The software aimed at the users of doctor-patient users and doctors; they have a certain basic knowledge of the computer and the ability to operate the computer. System maintainers are professionals in information management and information systems, familiar with operating systems and databases. They have the following characteristics:

The accuracy of information is high; the loss of false information or information will inevitably cause unnecessary loss.

The visiting time is short and the purpose of inquiry is strong.

The system relies on the functions of doctor end APP and patient end service APP in the health management system of traditional Chinese medicine (TCM) to support the objective

Through data center cloud storage and calculation analysis, the online Chinese health management realized. At the same time, the system can connect with the HIS system of hospital, solve the problem of isolated island of information in health management system, and realize the closed loop management of medical information.

\subsection{Development tools}

The system uses C / S architecture, all interfaces are simple and clear, the user interface described in detail in the functional requirements document. in an Integrated Environment using JAVA We use mysql5.6 database and maven3.2.5 library Apache activeMq to realize the platform design of synchronization between mobile phone APP and WeChat terminal.

\section{System total function design}

\subsection{Demand analysis}

According to the requirements of the hospital care center, the overall system function of the software is as follows:

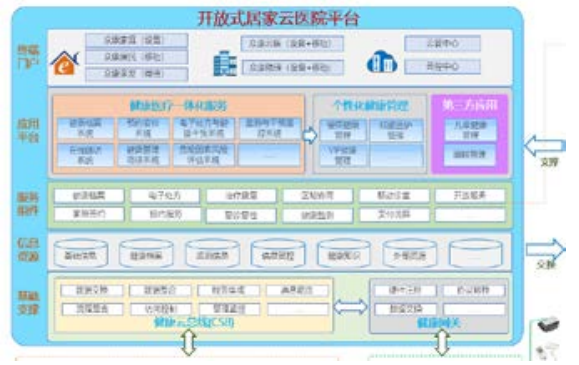

Fig. 1 Total function of health management system for end-care diseases

At the same time, the system is required to have its own characteristics, with the following functions:

1) Distinguish knowledge base for different disease population.

2) Doctors can monitor adverse drug reactions.

3) Make patient medication plan.

\subsection{Master processes and key tables}

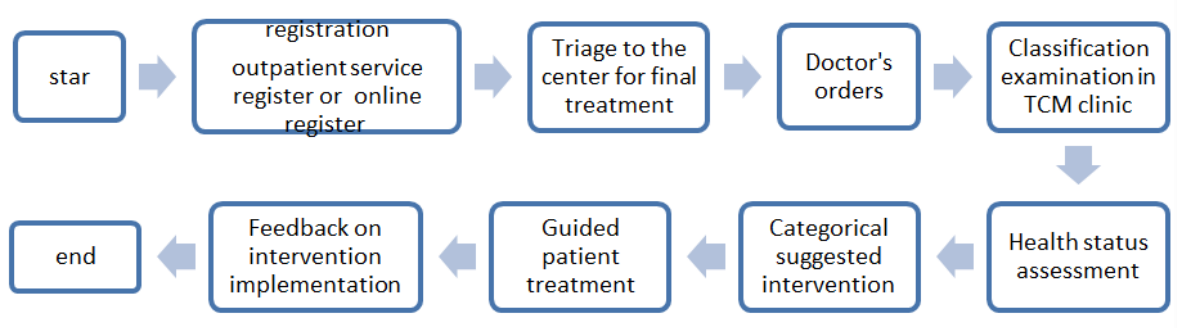

Fig.2 master processes 


\subsection{Introduction to key functions}

In the process of filing, there are several ways to file independent filing, fast filing, interface import and inviting registration. These methods can be convenient for patients and easy to connect with HIT system. The main index problem of hospital information system solved to the greatest extent. This is also a lot of HIT information management system did not consider the problem. At the same time, set the security authority, only the file personnel can operate and query the resident archives. In the file management interface, you can screen, sort and search residents, click on the button, you can display a certain type of residents according to the status, family, project and order information of the residents, or you can To be displayed according to an institution to which a resident belongs.
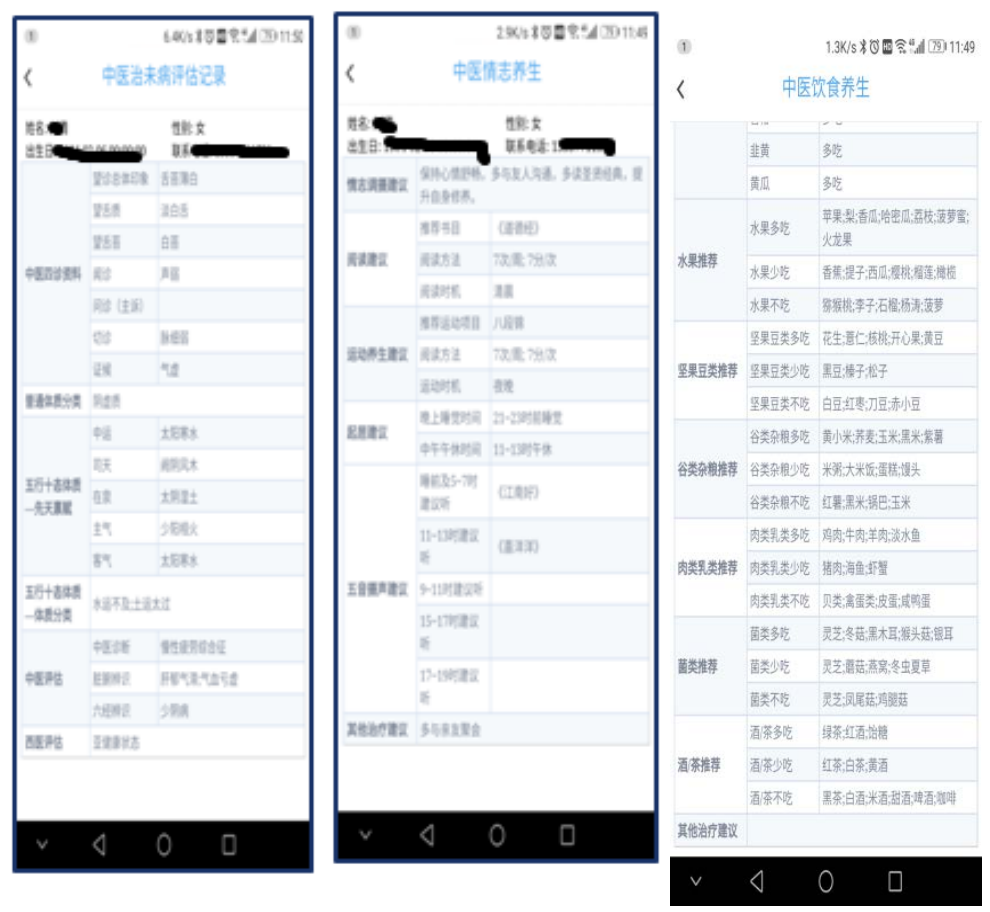

Fig.3 File management interface

Health care personnel can view or adjust the status of contracted residents, including termination of service, expiration of renewal, and so on. You can also check the signing details of residents, including the duration of service package, contract doctor and contract site photos.

Diagnostic service-TCM evaluation, completion of outpatient medical records, and these data can also extracted from the HIS, can also be linked to the hospitalization information system, arrangements for admission, post-hospital follow-up and other service functions.

Finally, the patient service evaluation-questionnaire feedback, for a single service special evaluation, doctors can at any time the patient evaluation, to improve the level of doctor service, timely tracking the patient's health status.

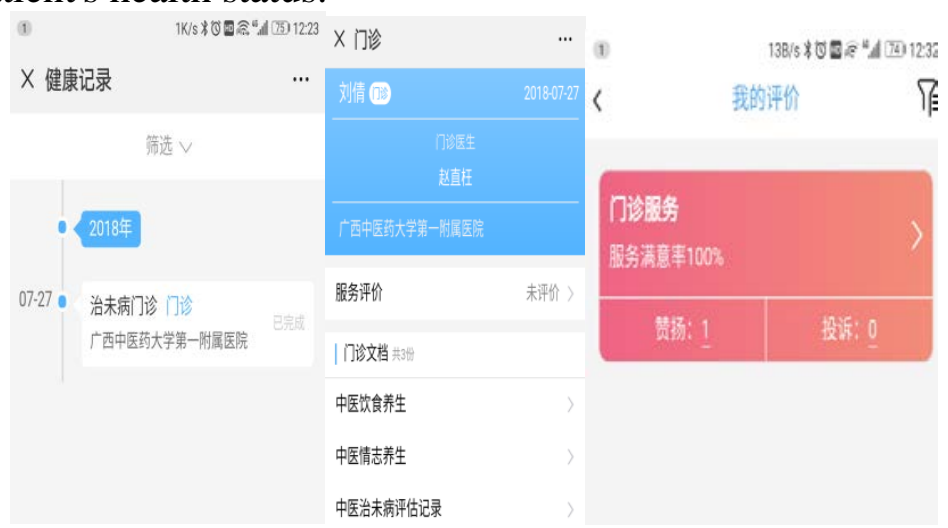

Fig.4 Signing details of residents 
In the self-health management module of patients, in addition to the patients according to the doctor's orders to treat, but also provides some articles and videos of health knowledge, so that patients can choose according to their actual situation. At the same time, the doctor can see the completion of the patient's orders. At the same time, a health assessment and analysis module made. According to the actual situation of the hospital, the questionnaire can make patients have a more professional understanding of their own health status; the basic table of the relationship is as follows:

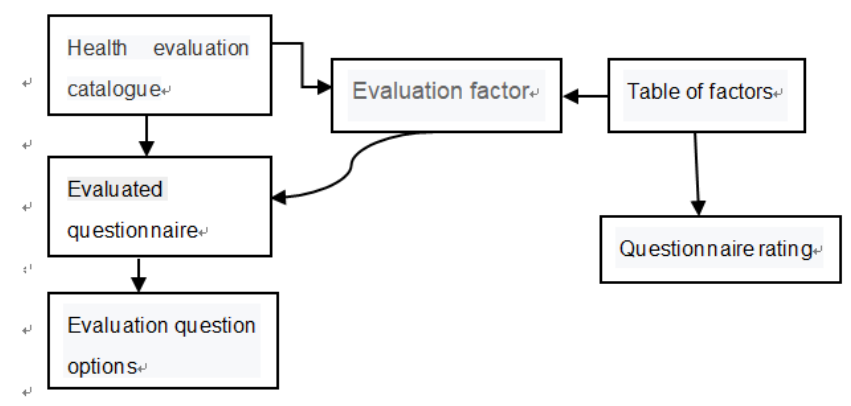

Fig. 5 Base table relation

All these data and treatment information data transmitted to the cloud server through the network, through data acquisition, to provide the basis for decision analysis and data mining.

\subsection{System features}

According to the usage habits of medical staff and people's operation norms, the system achieves the following usability requirements:

(1) At anytime the software on the mainframe or backup machine should have at least one that can used normally, and within a week, the software is not available on any computer for more than 10 percent of the total time.

(2) Moreover, be able to input the project must control; this is mainly for the background administrator;

(3) Software cannot appear more than 5 times in three days error.

(4) At the same time, when communicating with the patient, the patient can transmit five pictures to the server, which is helpful for doctors to identify TCM physique through pictures.

(5) Increase the problem of doctor-patient communication reply template, record the duration of the patient's execution, and make sure that the doctor can check whether the patient is performing daily tasks.

\section{Key technologies}

Cloud technology, which is based on "cloud computing" technology, refers to a kind of managed technology that unifies hardware, software, network and other resources in LAN or WAN to realize data computing, storage, processing and sharing. The system uses cloud technology to store huge data such as user's health data and TCM health knowledge into cloud health center. The system has good concurrency and stability. Users can quickly access the information in the database at any time, anywhere. At the same time, we use cloud computing to mine the user's health data, manage and analyze the user's data. Through the analysis of these data, we can make the health feedback information and individualized Chinese medicine training service for different users.

\section{Summary}

The system is divided into doctor interface and patient interface, the patient interface is simple and clear, easy to use, the doctor interface function is complete, the function has its own unique place, can connect with the HIT system, and complete the closed loop management of hospital information system. It is more suitable for medical service organizations to use. Based on the 
popular research of Android platform, the system has designed and implemented the management system of the end disease of TCM, and after many reconfigurations, testing and so on; this system has reached the desired research goal and can operated normally. The combination of traditional Chinese medicine (TCM) health care and traditional Chinese medicine (TCM) "preventing disease" can effectively improve the health of residents. Health level, expand the existing business of medical services, promote the rapid development of health services.

This is only a preliminary design. Because there is no unified and standardized hospital information system, it is necessary to integrate the data of different software together to form big data. It is still difficult to popularize and apply the project, and the interface of various kinds of software still needs to unify. This is a difficult problem in the research. We will concentrate technical personnel and personnel of the hospital information department. Community members do some work together.

\section{Acknowledgements}

Project: Guangxi science and technology planning project, project name: "Internet +" development and demonstration application of health management service cloud platform for Chinese medicine, and Project No.: 2017AB18056.

\section{References}

[1] Baidu encyclopedia: cure end disease word explanation, (ttps://baike.baidu.com/item/\%E6\%B2\%BB\%E6\%9C\%AB\%E7\%97\%85/129230)

[2] G G c, H C j, H K fa. Research and Development of traditional Chinese Medicine Health Care system based on a droid platform, Wireless Interconnection Technology. 2017 (22) 47: 49

[3] Z Yao, Chen S q, Wang L ting. Development and Application of Prevention and Health Care Management system for "treating and preventing Diseases" in online Chinese Medicine, Bright TCM 2016 (31) .2 Con 285287

[4] Jiang C j, Liu Z. Research on Mobile Application Development based on Cloud Computing [J]. Journal of Jiangsu Institute of Education (Natural Science) 2013 (4): 13-1

[5] F C s, Qin Z g, Yuan Ding. Cloud data security storage technology [J]. Journal of computer Science 2015 (1): 150-163 